\author{
Military Technical \\ College \\ Kobry El-Kobba \\ Cairo, Egypt
}

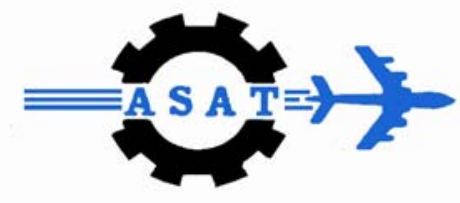

12-th International Conference

on

Aerospace Sciences \&

Aviation Technology

\title{
SHAPE OPTIMIZATION OF SHELL STRUCTURES
}

\author{
S. Y. Mahfouz*
}

\begin{abstract}
This paper deals with structural shape optimisation of prismatic shell structures using genetic algorithm. In the formulation of the optimisation problem, the minimum value of the strain energy is thought as objective function while the volume of each structure remains constant. The optimisation process is carried out for two structures: cylindrical and folded plate structures. The design variables are chosen such that the shape of each studied structure can be represented. The proposed algorithm, used to generate new structural shapes, is linked to a finite element package to calculate the objective function. It is observed that the proposed optimisation algorithm provides an efficient and reliable way of obtaining better solutions for such class of prismatic shell structures.
\end{abstract}

\section{KEYWORDS}

Genetic algorithm, Shape optimisation, Shell Structures

\section{INTRODUCTION}

The problem of shell optimisation has been of importance to structural engineers. Therefore, many authors developed algorithms to optimise the shape and size of shell structures. Shape and size optimisation of these structures using finite elements was investigated by Bletzinger and Ramm [1], Rao and Hinton [2], Afonso and Hinton [3], Ghasemi [4] and Lee [5]. Hinton et al [6] and Rao et al [7] also carried out shape and size optimisation of prismatic shell structures using the finite strip method. The main limitations using the FE models are the difficulty of handling branched shell structures. Further, the treatments of shape definition and mesh generation are complex. Moreover, the use of FE models is restricted by geometry and boundary condition limitations that makes the optimisation process time consuming.

The main task involved in the optimisation of any structure is to develop a suitable interface with an optimisation package, which is usually treated as a black box. An important step in the development of good models for the analysis and optimisation of shells is the introduction of an appropriate geometric model. In the initial definition of the shell, loads, boundary conditions and material disposition can be associated

\footnotetext{
* Egyptian Armed Forces
} 
with the geometric model. If a discrete analysis tool is to be used such as the FE method, then a suitable discretization may be produced using structured or unstructured meshing. Furthermore, if shape or size optimisation is to be carried out, then a suitable parameterization is required and design variables must be selected from the key data controlling the geometric model.

In the present paper the shape optimisation is performed with the aim to smooth the rough boundaries because of the difficulties to extract by the topology precise information on location and shape of the final boundaries. The paper also aims to develop an efficient and reliable procedure for the analysis and optimisation of shell structures using a simple to use algorithm based on modified genetic algorithm developed by Mahfouz and Raslan [8].

\section{GENETIC ALGORITHM}

Genetic algorithm (GA) is an optimisation strategy in which points in the design space are analogous to organisms involved in a process of natural selection. The term genetic is used because, along with the expected design representation, GA employs a code representation of design attributes that is analogous to a chromosome (see, Holland [9], Goldberg [10] and Davis [11]). This code is commonly a character string, with each character position being analogous to a gene, and each character assigned to a position being analogous to an allele. Organisms are generated and tested in generations, with offspring designs arising from parent designs. The creation of new designs for a new generation occurs with a process that is analogous to biological reproduction. Genetic crossover allows offspring designs to retain traits from parent designs, and infrequent mutations possibly yield radically improved designs. The testing of new designs is done with merit function, usually tailored to take the coded representation as input. In a given generation, designs with a higher merit are given a higher probability of creating offspring, and perhaps surviving themselves into the next generation.

Optimisation occurs, therefore, through a process of natural selection. Designs in a given generation group in pairs (i.e., mate), with the better designs having a higher probability of pairing. These parent designs produce offspring by genetic crossover. In single point crossover, a point along the coded representations (the chromosomes) is chosen at random, and the segments of the code after the point are swapped. After that random mutations are performed on individual alleles within the chromosomes by changing the values. These operations yield two new codes which represent two new designs that possess traits from both parents. The process then iterates. After many generations, the best design is achieved, because the merit function is more likely to allow better designs to produce offspring. Generally, the GA is judged to be successful if it evolves a population of highly fit individuals. Among many authors, Haftka, and Gurdal [12] and Michalewicz [13] may be consulted for more details regarding the various aspects of genetic algorithms.

The main features of the suggested modifications follow the basic assumption of a GA: the probability of getting fit children of two highly fit parents is higher than when one of the parents has a poor fitness. Accordingly, attempts have been made to accelerate obtaining population of a better average fitness thus reducing a chance of selection of poorly fit partner in the crossover operator. At first, the new technique uses a considerably larger population size before the actual start of the genetic 
algorithm. This initial large population contains $\left(N_{p o}=n N_{p}\right)$ randomly selected strings where $N_{p}$ is the size of all subsequent populations and $\mathrm{n}$ is a number of the order of ten. The fitness of all strings in this initial population is evaluated and $N_{p}$ best strings are selected to start the usual genetic operations. Obviously, in terms of computational effort this is equivalent to adding $\mathrm{n}$ iterations to the standard algorithm but, as the first regular population contains better strings, the modified algorithm tends to converge faster thus reducing the overall computational effort. At second, the new technique kills the worst individuals with a certain percentage, i.e. specifies how the less fit members are removed from the population. When using the traditional simple GA, it has been observed that the best individual of the population may fail to produce offspring for the next generation. The elitist strategy developed keeps the best individuals with a certain percentage termed elite ratio $E_{\mathrm{r}}$ of the population. Those individuals are called elite. The developed elitist strategy can be described as follows:

Step 1: Preparation of the data files, which includes GA parameters population size $N_{\mathrm{p}}$, elite ratio $E_{\mathrm{r}}$, probability of crossover $P_{\mathrm{c}}$, probability of mutation $P_{\mathrm{m}}$, crossover operator required and seed number.

Step 2: Creation of population with number of individuals equals to $N_{\mathrm{p}}$.

Step 3: Calculation of the objective function $F_{i}\left(\mathrm{i}=1,2, \ldots, N_{\mathrm{p}}\right)$ for each individual.

Step 4: Check of the feasibility of each individual using the predefined constraints.

Step 5: Computation of the value of the penalised objective function $P F_{i}$.

Step 6: Searching of the smallest $P F_{\text {best }}$ and largest $P F_{\text {worst }}$ value of the penalised objective function out of whole population $N_{\mathrm{p}}$.

Step 7: Evaluation of the fitness function $\left(F F_{i}\right)$ for all individuals:

$$
F F_{i}=P F_{\text {best }}+P F_{\text {worst }}-P F_{i} .
$$

Step 8: Sorting the whole population $N_{\mathrm{p}}$ according to the value of fitness function $\left(F F_{i}\right)$ of each individual where the largest value of $F F_{i}$ is the best.

Step 9: Killing the individuals $\left(N_{w}\right)$ having low fitness according to the week percentage $W_{p}$ of the population $\left(N_{\mathrm{p}}\right)$ where

$$
N_{w}=W_{p} \times N_{\mathrm{p}} .
$$

Step 10: In the surviving part $N_{\text {sur }}$, finding of the new value of the largest penalised objective function $P F_{\text {worst }}^{\text {new }}$ which is the worst values of the surviving part.

Step 11: Defining a new fitness function $F F_{i}{ }^{\text {new }}$ for only the surviving part $N_{\text {sur }}$ of the population. The fitness function $F F_{i}^{\text {new }}$ is

$$
F F_{i}^{\text {new }}=P F_{\text {best }}+P F_{\text {worst }}^{\text {new }}-P F_{i} .
$$

Step 12: Calculation of the probability of selection $P_{i}^{\text {sel }}$ of all the surviving individuals using 


$$
P_{i}^{\text {sel }}=\frac{F F_{i}^{\text {new }}}{\sum_{j=1}^{N_{\mathrm{s}}} F F_{j}^{\text {new }}} .
$$

Step 13: Filling in the new population. Here, the new population consists of: (1) part 1 contains the elite individuals. The number of these individuals $N_{\mathrm{e}}$

$$
N_{\mathrm{e}}=E_{\mathrm{r}} N_{\mathrm{p}}
$$

and it is filled by copying the best individuals out of the current population,

(2) part 2 contains the number of individuals $\left(N_{\mathrm{c}}\right)$ after crossover, where

$$
N_{\mathrm{c}}=P_{\mathrm{c}} N_{\mathrm{p}} \text {. }
$$

and it is filled by selecting its individuals according to the probability of selection discussed in step 12 and crossover the parents.

(3) part 3, the rest of population, whose number of individuals $\left(N_{\mathrm{r}}\right)$ is computed from

$$
N_{\mathrm{r}}=\left\{\begin{array}{ll}
N_{\mathrm{p}}-\left(N_{\mathrm{e}}+N_{\mathrm{c}}\right) & \text { if } P_{\mathrm{c}}+E_{\mathrm{r}}<1 \\
0 & \text { if } P_{\mathrm{c}}+E_{\mathrm{r}}=1
\end{array}\right. \text {. }
$$

and it is filled by randomly selecting its individuals from surviving $N_{\text {sur }}$ part.

Step 14: Check of the termination condition. In the present study, three termination conditions are used and if any of them is satisfied, then the process will terminate. These conditions are:

If the fittest design has not changed for 30 successive generations, or if the difference between the fittest $F^{\mathrm{cu}}$ of the current generation and that of 30 generations before is very small value $C^{\mathrm{cu}}$. This could be expressed in the form:

$$
\frac{F^{\mathrm{cu}}-F^{\mathrm{cu}-30}}{F^{\mathrm{cu}}} \leq C^{\mathrm{cu}}
$$

As proceeding with more generation, the population gets filled by more fit individuals, with perhaps a very small deviation from the fitness of the best individuals. Consequently, the average fitness comes very close to the fitness of the best design. This could result in another convergence criterion such that the percentage difference between the average fitness $F^{\text {av }}$ of the current population and the current fitness of the best design $F^{\mathrm{cu}}$ reaches a very small value $C^{\mathrm{av}}$. This can be expressed by:

$$
\frac{F^{\mathrm{cu}}-F^{\mathrm{av}}}{F^{\mathrm{cu}}} \leq C^{\mathrm{av}}
$$

When a total allocated number of generations $\left(\right.$ gen $\left.^{\max }=300\right)$ are reached.

If the conversion is satisfactory, then stop the program otherwise continue.

Step 15: Performing the mutation. The number of binary digits $N U_{d}$ that are changed can be computed from 


$$
N U_{\mathrm{d}}=N_{\mathrm{p}} P_{\mathrm{m}} \ell_{n_{\mathrm{v}}}^{\mathrm{T}}
$$

where $\ell_{n_{\mathrm{v}}}^{\mathrm{T}}$ is the number of chromosomes (string length) of one individual, and $P_{\mathrm{m}}$ is the probability of mutation.

Step 16: The process is repeated from Steps 3-14 until a converged solution is obtained, or a prescribed maximum number of iterations have been performed.

\section{DESCRIPTION OF THE PROFILE AND MESH GENERATION}

Among the various types of curves used for representing a shape, cubic splines are the most popular. The cubic spline meets the needs of most problems arising in practical applications. The profile of a typical prismatic shell is formed by an assembly of segments (Figure 1). Each segment is formed by an assembly of subsegments passing through certain key points all of which lie on the mid-surface of the shell. Each sub-segment is a cubic spline curve and spans between two adjacent key points within a segment. Some key points are common to different segments at their points of intersection. It is to be noted that the coordinates and thickness values of the key points are taken as design variables. The use of the coordinates and thicknesses at key points as design variables leads to fewer design variables and more freedom for controlling the shape of the shell.

Once the shape of the structure is defined in the transverse direction, the shape in the longitudinal directions can be defined by a range of mathematical functions to suit the geometric needs of the structure. For example, engineering structures such as plates, shells, tunnels and box girder bridges may have constant geometrical properties in the longitudinal or curvilinear direction.

Initially a mesh in the transverse direction forms the shell profile. This is usually carried out using an unstructured meshing procedure. This is sufficient to define the model (with a few additional single parameters such as length). Next, it is necessary to define the mesh in the longitudinal or spanning direction. In order to control the mesh density or spatial distribution of element sizes throughout the region of interest, the mesh density is specified at a sequence of key points in the shell structure. At the beginning of analysis, the mesh density values are given at the two end points of each segment. Based on the prescribed mesh density, the profile of the shell is discretized into a series of straight linear, quadratic or cubic elements. This mesh can be directly used for analysis. The transverse co-ordinates remain the same for FE analysis. The spanning co-ordinate of the nodes corresponds to the location of the section along the length of the prismatic shell. Further, for branched shells such as folded plates, no extra effort is required. The input data to the mesh generator is minimal and generates meshes of different sizes and types as specified. Figure 2 shows some examples of mesh generation on transverse profile and along the spanning direction for prismatic shells. 
In this section, the shape optimisation of classic cylindrical shell roof structure (see, Scordelis and Lo [14]) is investigated. The geometry of the structure is given in Figure 4 where $L=50.0 \mathrm{ft}$., $t=0.25 \mathrm{ft}$., $R=25.0 \mathrm{ft}$. The objective of optimisation is to minimize the strain energy (SE) of the structure with a constraint that the volume of the structure remains constant. The structure is modelled using 4 key points and one segment. Shape design variables at these key points are considered. The following material properties of the structure are assumed: The modulus of elasticity equals $4.32 \times 10^{8} \mathrm{lbs} / \mathrm{ft}^{2}$ and Poisson's ratio $v=0.0$. The cylindrical shell roof is subjected to a self weight loading of $80 \mathrm{lbs} / \mathrm{ft}^{2}$. The upper and lower limit of the design variables are given in Table 1 . Using a population size of 60 , single point crossover and probability of mutation equals 0.005 , the optimisation process is carried out. The termination conditions of the optimisation process are illustrated by Mahfouz [15]. The best solution is obtained after 147 iterations. Table 1 gives the values of the design variables at the optimum solution and Table 2 gives the values of strain energy (SE) and maximum displacement. It is observed that there is a reduction of nearly $54 \%$ in the SE and $85 \%$ in the maximum displacement values in the optimum structure compared to the initial structure. The initial and optimum shapes are also shown in Figure 3.

\section{SHAPE OPTIMIZATION OF FOLDED PLATE ROOF STRUCTURE}

The folded plate structure (Fries-Skene and Scordelis, [16]) shown in Figure 4 is studied. The initial thickness is considered to be same for all the plates. The geometric dimensions are chosen such that the length of the structure in the transverse direction is $70.0 \mathrm{ft}, \mathrm{t}=0.25 \mathrm{ft}, \mathrm{a}=8.67 \mathrm{ft}, \mathrm{b}=9.83 \mathrm{ft}, \mathrm{d}=5.0 \mathrm{ft}$, e = $=1.75$ $f t$ and the initial value of $S 1=3.0 \mathrm{ft}$. The modulus of elasticity equals $4.32 \times 10^{8}$ $\mathrm{lbs} / \mathrm{ft}^{2}$ and Poisson's ratio $v=0.0$. The structure is modelled using 4 key points and 3 segments. Both shape and thickness design variables are considered. The shell is subjected to a self weight loading of intensity $90 \mathrm{lbs} / \mathrm{ft}^{2}$. The objective of optimisation is to minimize the strain energy (SE) of the structure with a constraint that the volume of the structure remains constant. The upper and lower limit of the design variables are given in Table 3. Using a population size of 100 , single point crossover and probability of mutation equals 0.005 , the optimization process is carried out. The best solution is obtained after 161 iterations.

The initial and optimum shapes are shown in Figure 4. It is observed that there is a reduction of nearly $44 \%$ in the SE and $71 \%$ in the maximum displacement values in the optimum structure compared to the initial structure even for slight changes in the plate configurations. Table 3 gives the values of design variables and Table 4 gives the values of the SE and maximum displacement.

\section{CONCLUSION}

The methodology of shape optimization using genetic algorithm is suggested. The optimization procedure based on genetic algorithm is reliable as considerable reduction in strain energy and maximum displacement can be observed. The tools developed can also be used for designing novel structural forms. The present work can be easily extended to shells with curved and skew plan-form subjected to multiload cases and multi-criteria optimization. 


\section{REFERENCES}

[1] Bletzinger, K.U. and Ramm, E. (1993), "Form finding of shells by structural optimization", Engineering with Computers, Vol 9, pp. 27-35.

[2] Rao, N.V.R. and Hinton, E. (1993), "Structural optimization of variable thickness plates and free form shells", Structural Engineering Review , Vol 5, pp. 1-21.

[3] Afonso, S.M.B. and Hinton, E. (1995), "Free vibration analysis and shape optimization of variable thickness plates and shells-II, sensitivity analysis and shape optimization", Structural Engineering Review ,Vol. 10, pp. 47-66.

[4] Ghasemi, M. R. (1996), "Structural Optimization of Trusses and Axisymmetric Shells Using Gradient-Based Methods and Genetic Algorithm", Ph.D thesis, University of Wales, Swansea, UK.

[5] Lee, S.J.(1998), Analysis and optimization of shells, Ph.D. Thesis, Dept. of Civil Engg., Univ. Col.. of Swansea.

[6] Hinton, E. Ozakca, M. and Rao N.V.R. (1995), "Free vibration analysis and shape optimisation of variable thickness prismatic folded plates and curved shells, Part 1 and 2", J.Sound and Vibration, Vol 181, pp.567-581.

[7] Rao, N.V.R., and Hinton, E. (1994), "Analysis and optimisation of prismatic plate and shell structures with curved planform, Part 2 --- shape optimisation", Computers and Structures, Vol. 52, pp. 341-351.

[8] Mahfouz, S. Y. and Raslan , M. S. , (2004) " Structural shape optimization using modified genetic algorithms ", International conference on Civil and Arch, Military Technical College, Cairo, Egypt, Vol. , pp.

[9] Holland, J.H., "Adaptation in Natural and Artificial Systems", University of Michigan, Ann Arbor, 1975.

[10] Goldberg, D.E. (1989), "Genetic Algorithms in Search, Optimization and Machine Learning", Addison-Wesley, Reading, MA.

[11] Davis, L. (1991), "Handbook of Genetic Algorithms", Van Nostrand Reinhold, New York.

[12] Haftka, R. T. and Gurdal, Z., "Elements of Structural Optimization", Kluwer Academic Publishers, London, 1993.

[13] Michalewicz, Z. (1996), "Genetic Algorithm + Data Structures = Evolution Programs", 3rd ed., Springer-Verlag, New

York.

[14] Scordelis, A.C. and Lo, K.S. (1969), "Computer analysis of cylindrical shells", ACl journal, Vol 61, pp. 539-561.

[15] Mahfouz, S. Y., (2002) " Maximization of the ratio of the effective buckling length Evaluated by FEM and BS5950", $4^{\text {th }}$ International Conference on Civil and Architecture Engineering, MTC, Cairo, Egypt.

[16] De Fries-Skene, A., and Scordelis, A.C. (1964), "Direct solution of folded plates", Journal of Structural Division, ASCE, pp. 15-47. 

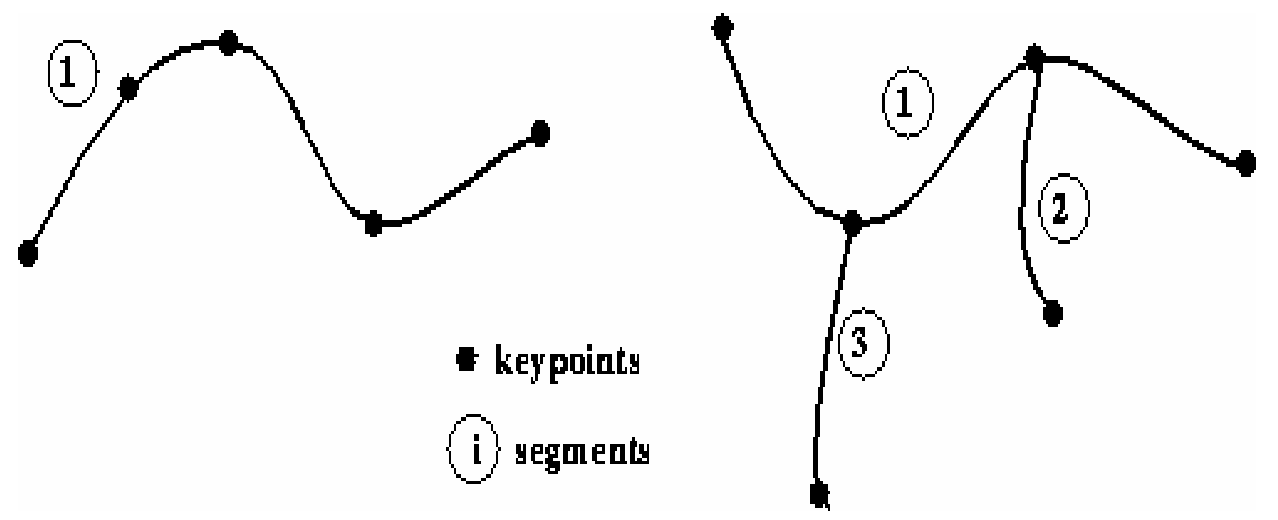

Figure 1 Representation of structural cross-sectional geometry of smooth and branched prismatic shells
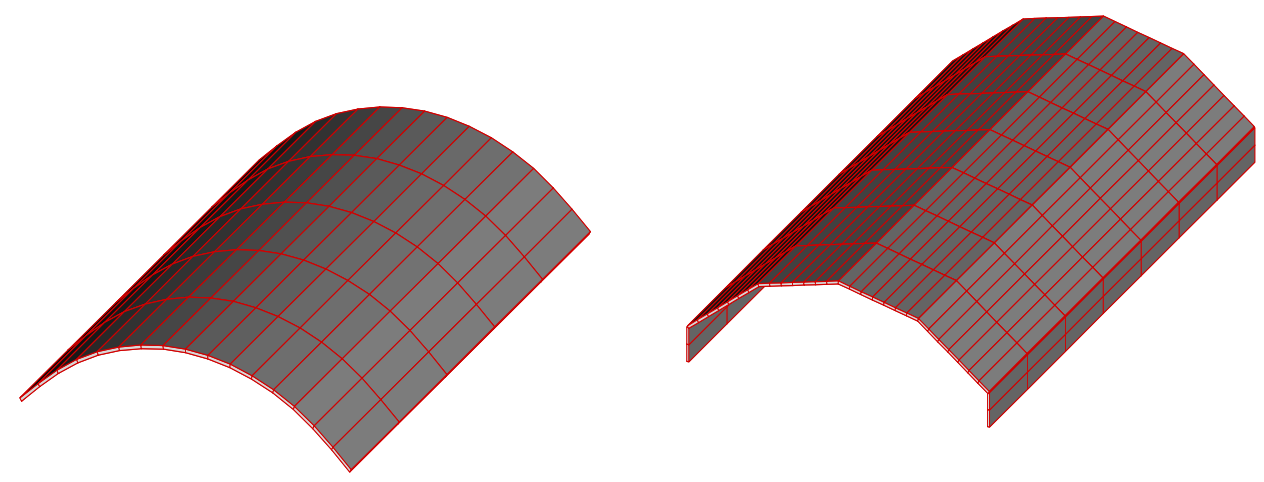

Figure 2 Mesh generation on the transverse profile and along the spanning direction of shells 


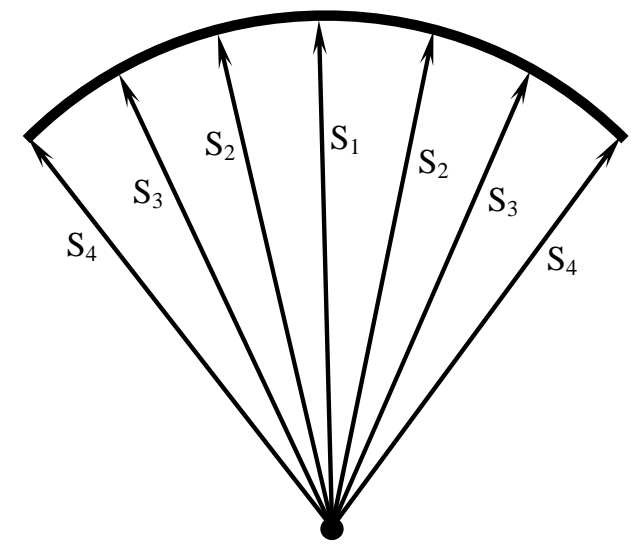

a) shape design variables

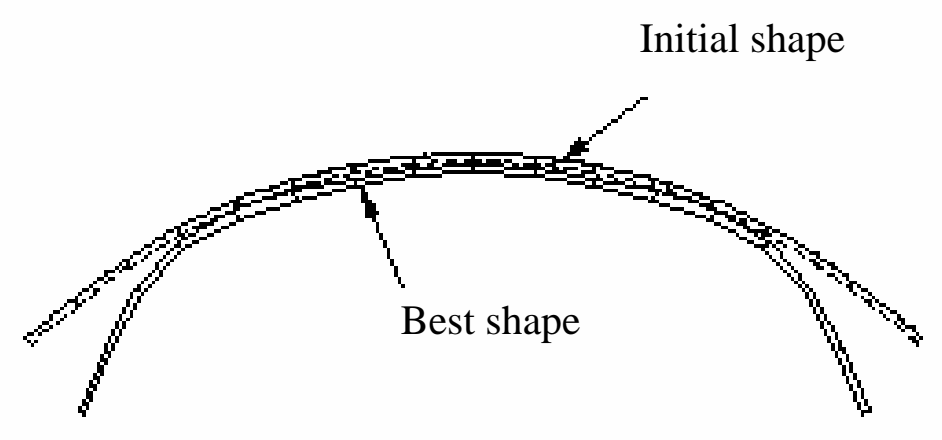

(b) Initial and best shape

Figure 3 Cylindrical shell roof

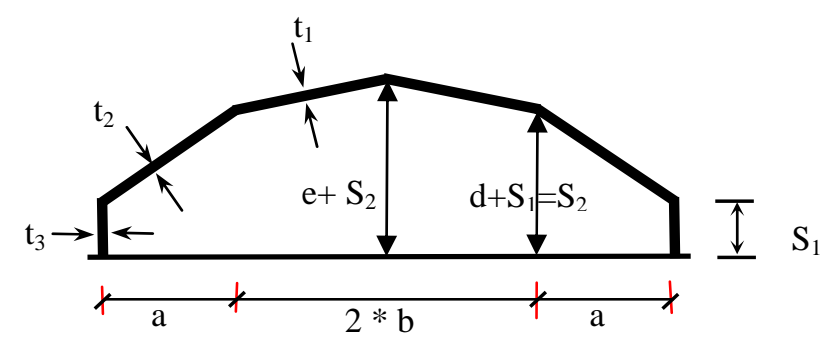

(a) Structural geometry best shape

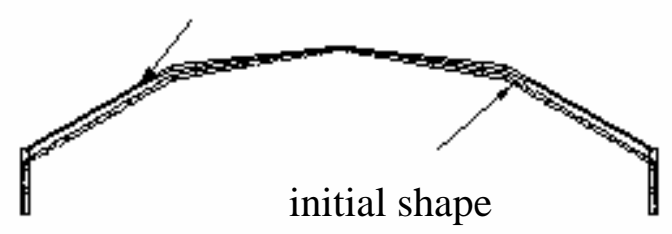

(b) Initial and best shape

Figure 4 Folded plate structure 
Table 1 Cylindrical shell roof structure: values of the design variables.

\begin{tabular}{|c|c|c|c|}
\hline $\begin{array}{c}\text { Design } \\
\text { variables }\end{array}$ & $\begin{array}{c}\text { Lower } \\
\text { limit }\end{array}$ & $\begin{array}{c}\text { Upper } \\
\text { limit }\end{array}$ & Obtained values \\
\hline$s_{1}$ & 17.0 & 28.0 & 22.0 \\
\hline$s_{2}$ & 17.0 & 28.0 & 24.5 \\
\hline$s_{3}$ & 17.0 & 28.0 & 24.6 \\
\hline$s_{4}$ & 17.0 & 28.0 & 24.6 \\
\hline
\end{tabular}

Table 2 Cylindrical shell roof structure: initial and optimal values of strain energy and maximum displacement.

\begin{tabular}{|c|l|l|l|l|}
\hline \multirow{2}{*}{ Method } & \multicolumn{2}{|c|}{ Strain energy } & \multicolumn{2}{c|}{ Max. displacement } \\
\cline { 2 - 5 } & Initial & Obtained & Initial & Obtained \\
\hline FE & 9076 & 4148 & 0.290 & 0.037 \\
\hline
\end{tabular}

Table 3 Folded plate roof structure: values of the design variables.

Table

\begin{tabular}{|c|c|c|c|}
\hline $\begin{array}{c}\text { Design } \\
\text { variables }\end{array}$ & $\begin{array}{c}\text { Lower } \\
\text { limit }\end{array}$ & $\begin{array}{c}\text { Upper } \\
\text { limit }\end{array}$ & Obtained values \\
\hline$s_{1}$ & 3.00 & 9.75 & 8.58 \\
\hline$s_{2}$ & 1.00 & 8.00 & 3.76 \\
\hline$t_{1}$ & 0.10 & 0.50 & 0.20 \\
\hline$t_{2}$ & 0.10 & 0.50 & 0.20 \\
\hline$t_{3}$ & 0.10 & 0.50 & 0.47 \\
\hline
\end{tabular}

Folded plate roof structure: initial and optimal values of strain energy and maximum displacement.

\begin{tabular}{|l|l|l|l|l|}
\hline \multirow{2}{*}{ Method } & \multicolumn{2}{|c|}{ Strain energy } & \multicolumn{2}{c|}{ Max. displacement } \\
\cline { 2 - 5 } & Initial & Obtained & Initial & Obtained \\
\hline FE & 11593 & 6468 & 0.155 & 0.045 \\
\hline
\end{tabular}

